\title{
A chemotherapy-free regimen for Philadelphia chromosome-positive acute lymphoblastic leukemia: are we there yet?
}

\author{
Yishai Ofran ${ }^{1,2}$
}

${ }^{1}$ Department of Hematology and Bone Marrow Transplantation, Rambam Health Care Campus, and ${ }^{2}$ The Ruth and Bruce Rappaport Faculty of Medicine, Technion, Haifa, Israel

E-mail: YISHAI OFRAN - y_ofran@rambam.health.gov.il

doi:10.3324/haematol.2020.278077

$\mathrm{N}$ ovel therapies are revolutionizing the treatment strategies for Philadelphia chromosome-positive $(\mathrm{Ph}+)$ acute lymphoblastic leukemia (ALL). Ten years ago, the GIMEMA (Gruppo Italiano Malattie EMatologiche dell'Adulto) ALL Working Party pioneered a chemotherapyfree induction regimen using the dasatinib-steroid combination, ${ }^{1}$ which brought about complete hematological remission (CHR) in all 53 evaluable patients ${ }^{2}$ and complete molecular response (CMR) in ten of them (18.8\%).

In this issue, final results of a subsequent LAL1509GIMEMA prospective single-arm trial are reported. ${ }^{3}$ The treatment protocol included 1-month induction with the aforementioned combination, followed by dasatinib extension until day 85 . The post-remission regimen was assigned based on the minimal residual disease (MRD) status. Of the 60 enrolled patients (median age 41.9 years [range, 18-60]), those who achieved CMR were subject to dasatinib maintenance with no further intensification. The majority of patients (47 of $60 ; 78 \%$ ) achieved CHR, while testing MRDpositive post-induction. These patients were assigned to allogeneic stem cell transplantation (allo-SCT) with or without consolidation chemotherapy. Patients ineligible for transplantation were consolidated with chemotherapy only. No induction deaths were reported and the CHR rate by day 85 was $97 \%$, with CMR achieved in 11 of $60(18.3 \%)$ patients. At a median follow-up of almost 5 years, overall survival (OS) and disease-free survival (DFS) were $56.3 \%$ and $47.2 \%$, respectively.

Are these impressive data sufficient to set the stage for a new standard of care in Ph+ALL? One of the critical achievements of these two studies, that should be taken into consideration, is the absence of induction deaths among the 113 patients treated. To that end, future $\mathrm{Ph}+\mathrm{ALL}$ treatment protocols should be designed with the aim to maintain such a minimal induction-related mortality rate. However, this attractive low-intensity induction regimen may be unsuitable for higher-risk Ph+ALL patients. In the LAL1509GIMEMA protocol, the post-induction MRD status has been the sole factor used to stratify patients for intensive consolidation followed by allo-SCT versus dasatinib maintenance only. Yet, the clinical significance of MRD results depends on a variety of patient- and treatment-related parameters.

Based on data from previous ALL studies, the Food and Drug Admisnsitration has accepted an MRD level of less than $0.01 \%$ as a surrogate efficacy endpoint for new drugs in ALL. ${ }^{4}$ Surprisingly and disappointingly, in the current trial, four of $11(36 \%)$ patients who achieved CMR with the dasatinib-steroid induction eventually relapsed. Three of these relapses were diagnosed early at a molecular level and therefore never fulfilled the former criteria of relapse. This must raise a red flag and a message regarding the complexity of $M R D$ clinical interpretation should be played out loud. MRD negativity is not synonymous to cure but it is rather its biomarker. No matter how sensitive the available tests are, there is still room for residual disease presence at a level below the threshold of detection. Thus, ultimate degrees of disease eradication for patients who achieve MRD negativity following intensive and less intensive induction may be different. A negative MRD result following intensive induction reflects an even deeper response than the sensitivity cutoff of the test used. This should not be extrapolated to the outcome prediction following less intensive protocols when the actual level of response below the MRD negativity cutoff may be lower (Figure 1). Not only the specific induction protocol but also

\section{A} Proportion of
malignant cells

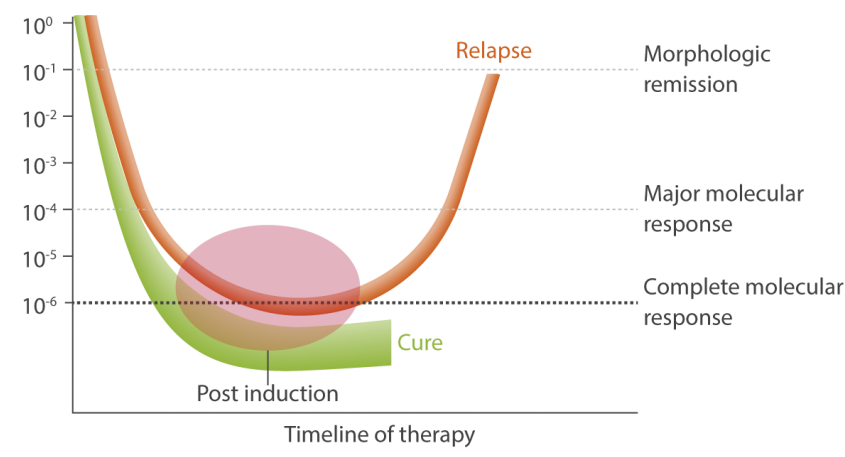

B Distribution of response levels after non-intensive induction

Proportion of

malignant cells

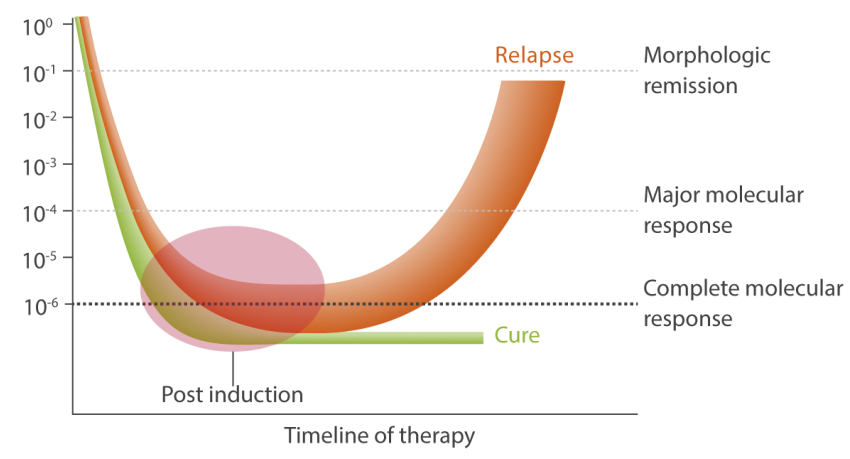

Figure 1. Response after intensive and non-intensive induction in a population of Philadelphia chromosome-positive acute lymphoblastic leukemia patients. (A) Distribution of response levels after intensive induction. (B) Distribution of response levels after non-intensive induction. The distribution of the actual depth of response across patients who achieved minimal residual disease negativity following intensive and non-intensive induction differs and so does the risk of relapse. 
the characteristics of the patient population should be taken into account. For instance, in Ph-negative ALL, achievement of identical laboratory major molecular response levels following treatment with intensive chemotherapy and tyrosine kinase inhibitors, was shown to predict considerably different relapse-free survival rates for newly diagnosed versus relapsed patients $(26.1 \mathrm{vs}$. 12 months, respectively), ${ }^{5,6}$ Notably, progression-free survival for relapsing patients who achieved MRD negativity with inotuzumab ozogamicin treatment was as short as 8.6 months. ${ }^{7}$ Thus, in different clinical settings, identical laboratory results may be associated with completely different predicted outcomes.

The LAL1509 trial, while demonstrating feasibility of a chemotherapy-free regimen for some patients, has also highlighted that biological differences within the Ph+ALL patient population go far beyond the presence or absence of $B C R / A B L$ mutations such as T315I. In this trial, patients presenting with genetic aberrations in IKZF1 plus CDKN2A/B and/or PAX5 $\left(I K Z F 1^{+}\right)$demonstrated a relapse-free survival rate of $0 \%$. This combination of genetic abnormalities is known to portend poor prognosis even in patients undergoing allo-SCT. Yet, the relapsefree survival rates reported in previous studies using intensive induction regimens have been better. ${ }^{8}$ Moreover, the GIMEMA group has lately reported results of using a still more attractive combination of steroids, dasatinib and blinatumomab, ${ }^{9}$ that has led to considerable improvement in the survival of all patients, including those presenting with $I K Z F 1^{+}$aberrations.

What have we learned from the current trial? First, induction death in $\mathrm{Ph}+\mathrm{ALL}$ is preventable and for some patients even non-intensive induction should be considered. Second, the way MRD negativity is achieved influences its clinical implications. Third, routine screening for $I K Z F 1^{+}$abnormalities is advised and may be considered when the intensity of an induction regimen is selected. And last but definitely not least, a combination of steroids, tyrosine kinase inhibitors and blinatumomab, given as first-line therapy, is an attractive option for
$\mathrm{Ph}+\mathrm{ALL}$ patients and is currently being studied as part of a large intergroup prospective phase III trial, led by ECOG-ACRIN, EA9181 (clinicaltrials gov. Identifier: NCT04530565). ${ }^{10}$

\section{Disclosures}

No conflicts of interest to disclose.

\section{References}

1. Vignetti M, Fazi P, Cimino G, et al. Imatinib plus steroids induces complete remissions and prolonged survival in elderly Philadelphia chromosome-positive patients with acute lymphoblastic leukemia without additional chemotherapy: results of the Gruppo Italiano Malattie Ematologiche dell'Adulto (GIMEMA) LAL0201-B protocol. Blood. 2007;109(9):3676-3678.

2. Foa R, Vitale A, Vignetti M, et al. Dasatinib as first-line treatment for adult patients with Philadelphia chromosome-positive acute lymphoblastic leukemia. Blood. 2011;118(25):6521-6528.

3. Chiaretti S, Ansuinelli M, Vitale A, et al. A multicenter total therapy strategy for de novo adult Philadelphia chromosome positive acute lymphoblastic leukemia patients. Final results of the GIMEMA LAL 1509 protocol. Haematologica. 2021;106(7):1828-1838.

4.FDA Guidance Document: Hematologic Malignancies: Regulatory Considerations for use of minimal residual disease in development of drug and biological products for treatment. Available at https://www.fda.gov/media/134605/download. Accessed in December, 2020.

5. Short NJ, Jabbour E, Sasaki K, et al. Impact of complete molecular response on survival in patients with Philadelphia chromosome-positive acute lymphoblastic leukemia. Blood. 2016;128(4):504-507.

6. Abou Dalle I, Kantarjian HM, Short NJ, et al. Philadelphia chromosome-positive acute lymphoblastic leukemia at first relapse in the era of tyrosine kinase inhibitors. Am J Hematol. 2019;94(12):13881395 .

7. Jabbour E, Gokbuget N, Advani A, et al. Impact of minimal residual disease status in patients with relapsed/refractory acute lymphoblastic leukemia treated with inotuzumab ozogamicin in the phase III INO-VATE trial. Leuk Res. 2020;88:106283.

8. Pfeifer H, Raum K, Markovic S, et al. Genomic CDKN2A/2B deletions in adult $\mathrm{Ph}(+)$ ALL are adverse despite allogeneic stem cell transplantation. Blood. 2018;131(13):1464-1475.

9. Foa R, Bassan R, Vitale A, et al. Dasatinib-Blinatumomab for Ph-positive acute lymphoblastic leukemia in adults. N Engl J Med. 2020; 383(17):1613-1623.

10. Testing the use of steroids and tyrosine kinase inhibitors with blinatumomab or chemotherapy for newly diagnosed BCR-ABL-positive acute lymphoblastic leukemia in adults. Available at https://clinicaltrials.gov/ct2/show/NCT04530565. Accessed in December, 2020. 